\title{
Ocular phenotype and electroretinogram abnormalities in Lafora disease
}

\author{
A "window to the brain"
}

Ajoy Vincent, MD, Angelo Macrì, MD, Anupreet Tumber, MSc, Nikolas Koukas, MD, Saija Ahonen, PhD, Pasquale Striano, MD, PhD, and Berge Minassian, MD

Neurology ${ }^{\circledR}$ 2018;91:137-139. doi:10.1212/WNL.0000000000005821

Lafora disease (LD) is a teenage-onset progressive neurologic disorder caused by biallelic mutations in EPM2A (laforin) or EPM2B (malin) encoding laforin phosphatase and malin ubiquitin E3 ligase, respectively, both involved in glycogen structural integrity. Defective laforin or malin results in neuronal accumulation of malformed insoluble glycogen termed Lafora bodies (LBs). ${ }^{1}$ Histologic reports indicate LB accumulation in the inner retinal layers with evidence of bipolar cell atrophy. ${ }^{2}$ Funduscopic retinal examination has been reported unremarkable except in one patient with unilateral optic atrophy that may have been unrelated to LD. ${ }^{3,4}$ Recently, retinitis pigmentosa was reported in a 21 -year-old patient diagnosed only on skin biopsy, and was highlighted on the journal cover. ${ }^{5}$ A subsequent letter to the editor suggested that this may represent an instance of false-positive interpretation of skin biopsy, but the debate remains unsettled. ${ }^{1,5,6} \mathrm{We}$ aimed to address this issue by performing multimodal imaging and electrophysiology (e-Methods in links.lww.com/WNL/A580) to characterize the eye phenotype in 4 patients with genetically confirmed LD, concurrently setting the stage to identify potentially useful ophthalmologic biomarkers in LD.

e-Methods and tables e-1 and e-2, links.lww.com/WNL/A578, summarize the genetic results and clinical phenotype of the patients.

\section{Patient 1}

This 21-year-old man (homozygous deletion of EPM2A exons 1 and 2) had first seizure episode at 14 years of age. Best-corrected visual acuity (BCVA) was 20/40 (right eye) and 20/25 (left eye). Retinal examination, fundus autofluorescence (FAF) imaging, and central retinal thickness and lamination were normal (figure, A-C). Full-field electroretinogram (ERG) testing showed rod bipolar cell impairment with mild cone system dysfunction (figure e-1, links.lww.com/WNL/A579).

\section{Patient 2}

A 22-year-old woman had first seizure at 14 years of age and harbored compound heterozygous EPM2A mutations (p.Asn163Asp/p.Ala254Metfs*33). BCVA was 20/40 bilaterally. Retinal examination, FAF, and central retinal thickness and lamination were normal (figure, D-F). The ERG showed mild cone system dysfunction; rod function was normal (figure e-1, links.lww.com/WNL/A579).

\section{Patient 3}

A 21-year-old woman with homozygous EPM2B missense mutations (p.Pro69Ala) had seizure onset at 14 years of age. BCVA was $20 / 25$ bilaterally with refractive correction of $-3.50 \mathrm{D}$

From the Hospital for Sick Children (A.V., A.T., S.A., B.M.), Toronto; University of Toronto (A.V.), Canada; Polyclinic Hospital San Martino (A.M.), Genova; University of Genova (N.K., P.S.); G. Gaslini Institute (P.S.), Genova; and University of Texas Southwestern (B.M.), Dallas.

Go to Neurology.org/N for full disclosures. Funding information and disclosures deemed relevant by the authors, if any, are provided at the end of the article.

\author{
Correspondence \\ Dr. Minassian \\ berge.minassian@sickkids.ca
}


Figure Retinal imaging findings in Lafora disease
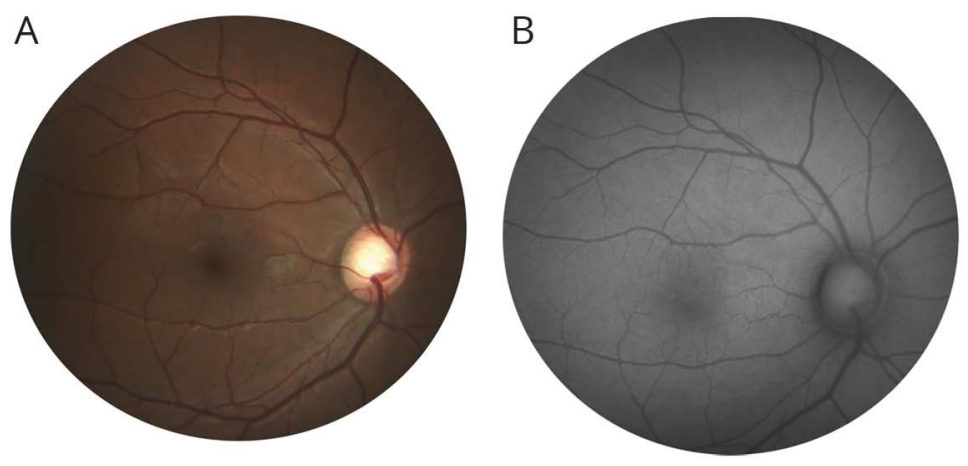

C
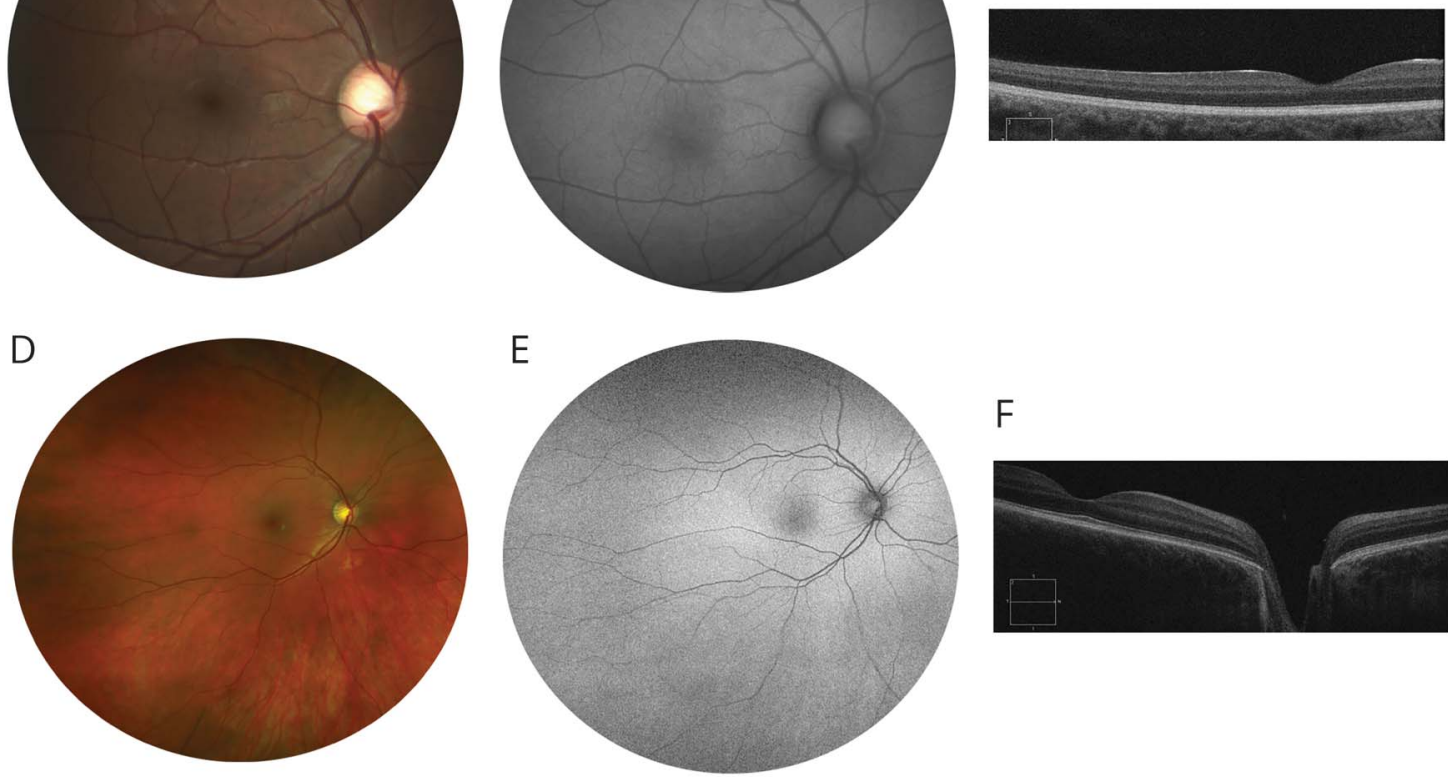

$\mathrm{F}$
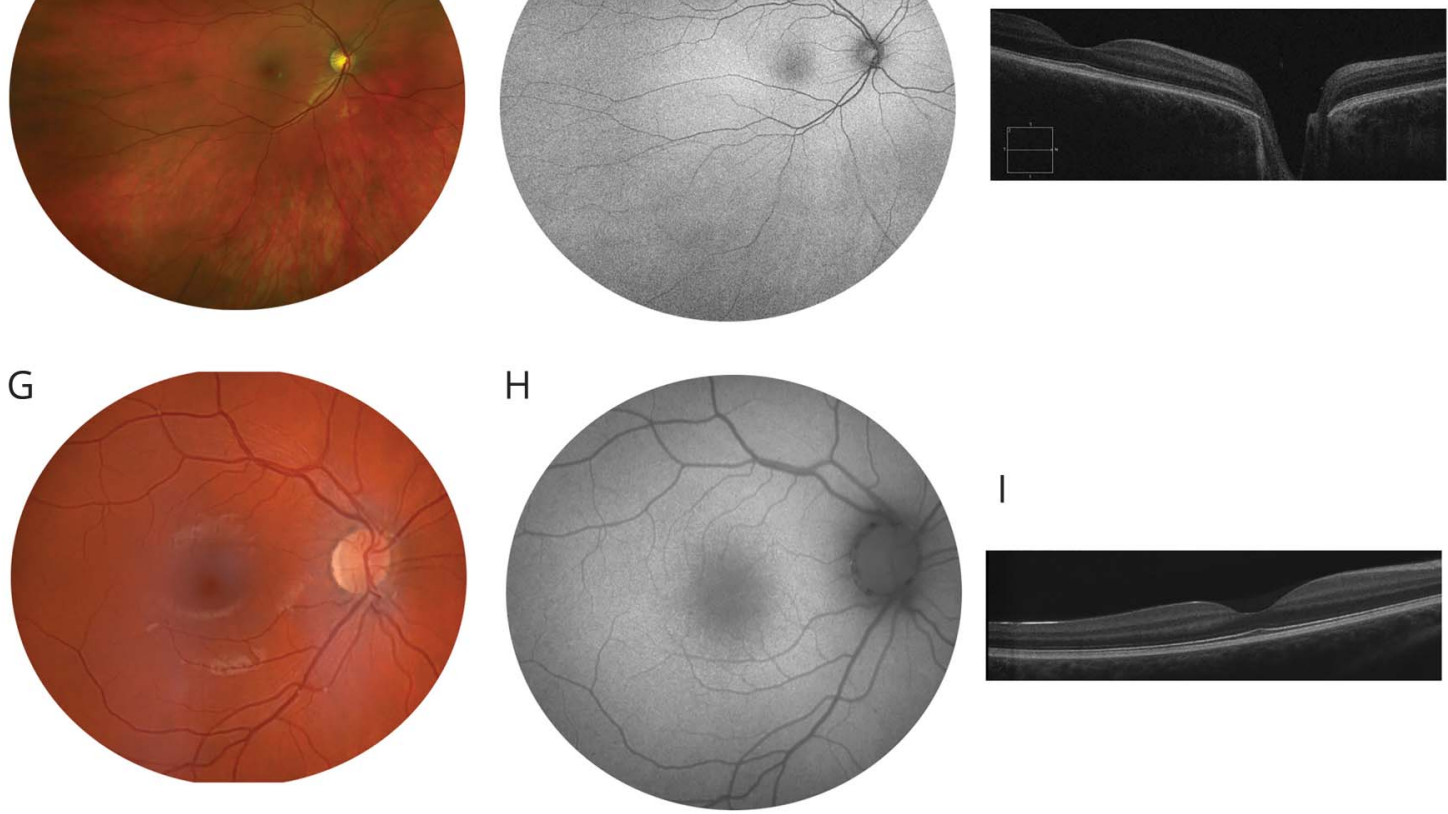

I
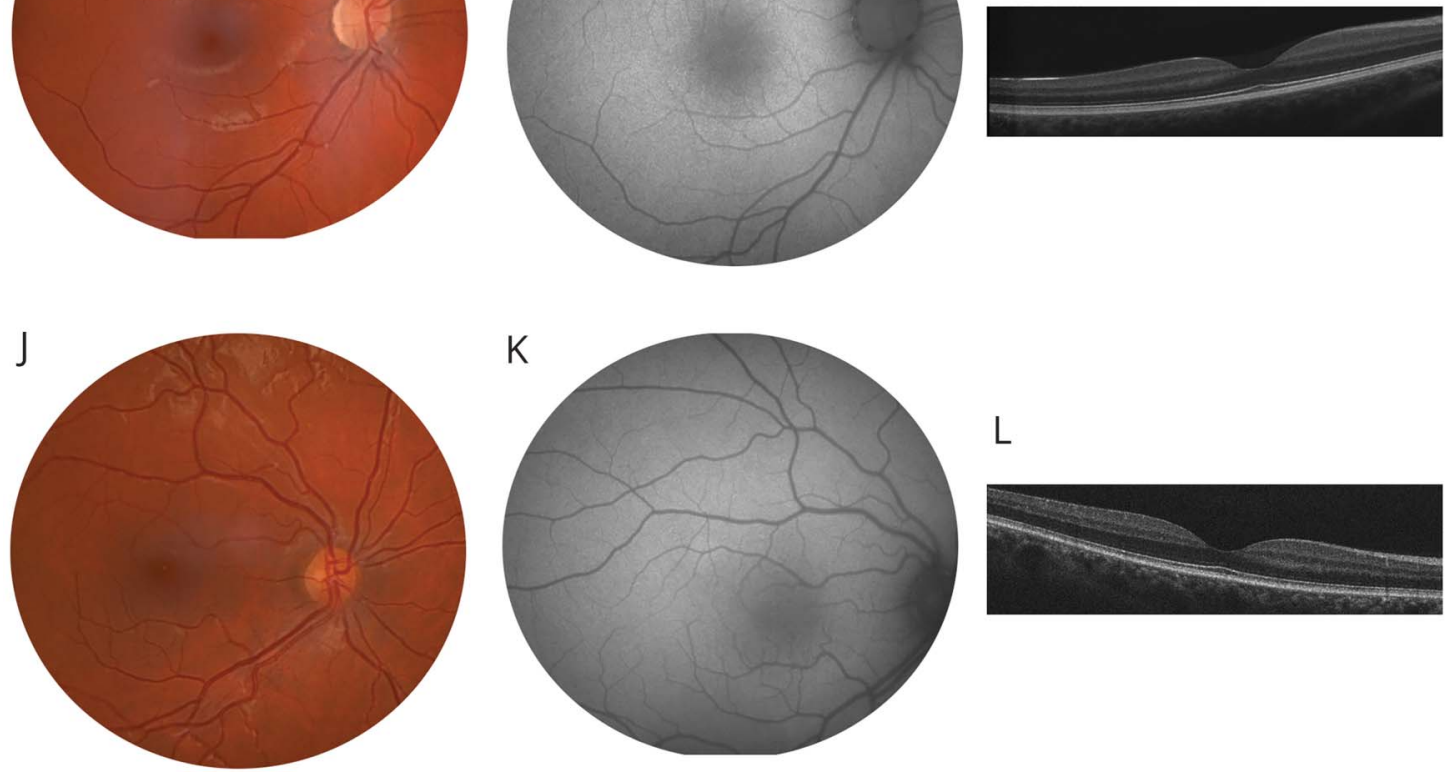

$\mathrm{L}$

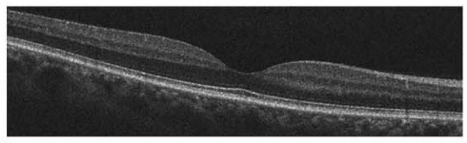

(A-C) Images from the right eye of patient 1. (A) Normal fundus photograph. (B) Normal fundus autofluorescence (FAF) image suggests normal lipofuscin levels in the retinal pigment epithelium. (C) Spectral-domain optical coherence tomography (SD-OCT) single line scan through the macula shows normal central retinal thickness and lamination. (D-F) Images from the right eye of patient 2 . Fundus and FAF show a wider field of the retina. As described in case 1 , fundus, FAF and SD-OCT (D-F) are normal. (G-I) Images from the right eye of patient 3. Fundus, FAF, and SD-OCT (G-I) are normal. (I-L) Images from the right eye of patient 4. Fundus, FAF, and SD-OCT (J-L) are normal. 
sphere. Retinal examination, FAF, and central retinal thickness and lamination were normal (figure, G-I). The ERG showed rod photoreceptor and bipolar cell impairment with additional cone system dysfunction (figure e-1, links.lww.com/WNL/A579).

\section{Patient 4}

A 31-year-old man with homozygous EPM2B missense mutations (p.Asp308Val) had seizure onset at 16 years. BCVA was $20 / 25$ in either eye with refractive correction of $-1.00 \mathrm{D}$ sphere. Retinal examination, FAF, and central retinal structure were normal (figure, J-L). The ERG showed rod photoreceptor and bipolar cell impairment with additional cone system dysfunction (figure e-1, links.lww.com/WNL/A579).

\section{Discussion}

Newer noninvasive in vivo retinal imaging techniques and electrodiagnostic testing better aid in disease diagnosis and characterization; notably, considering the eyes as windows to the brain, there is marked interest in in vivo retinal imaging in neurodegenerative disorders to identify biomarkers of early disease.

All our patients with LD demonstrated good visual acuity (20/40 or better) and normal retinal examination (including optic disc); none had retinitis pigmentosa. FAF, a noninvasive retinal imaging technique that provides in vivo density map of fluorophores in the retinal pigment epithelium, demonstrated normal pattern in all 4 patients; this observation excludes retinal pigment epithelial atrophy and any significant structural abnormality of the photoreceptor outer segments in the central retina $\left(50^{\circ}\right)$. We did not detect any structural alterations in the macula on spectral-domain optical coherence tomography (in vivo imaging that provides $\sim 5 \mu \mathrm{m}$ axial resolution); a prior histologic report observed LB deposition in inner retinal layers of macula. ${ }^{2}$

Our study revealed abnormal retinal function on ERG testing in all patients. Generalized rod bipolar cell dysfunction as evidenced by reduced b/a ratio of scotopic ERG was present in 3 , and generalized cone system dysfunction with cone inner retinal (bipolar) involvement was observed in all 4 patients. The bipolar cell dysfunction observed on ERG is in keeping with the histologic bipolar cell atrophy described in LD. ${ }^{2}$ The ERG also demonstrated generalized reduction in rod photoreceptor function (decreased rod a-wave amplitude) in both $E P M 2 B$ patients. Although no abnormality was noted in photoreceptor layers in postmortem eyes (genes unknown), the observed rod a-wave changes in $E P M 2 B$ patients may indicate functional changes to precede structural changes in specific disease stages or may be unique to EPM $2 B .{ }^{2}$ Reports using early ERG techniques (1978 or earlier) suggested normal responses or transiently subnormal bipolar cell activity in $\mathrm{LD}^{3,7}$; the current techniques are superior, and can better delineate the locus of abnormality.

ERG may be a valuable tool to monitor LD progression, and potentially serve as a useful biomarker to investigate intervention efficacy in gene replacement or other therapeutic clinical trials.

\section{Author contributions}

Ajoy Vincent: study concept and design, acquisition of data, analysis and interpretation, wrote the draft manuscript. Angelo Macrì: acquisition of data, critical revision of the manuscript for important intellectual content. Anupreet Tumber: acquisition of data, critical revision of the manuscript for important intellectual content. Nikolas Koukas: acquisition of data, critical revision of the manuscript for important intellectual content. Saija Ahonen: analysis and interpretation, critical revision of the manuscript for important intellectual content. Pasquale Striano: acquisition of data, critical revision of the manuscript for important intellectual content. Berge Minassian: study concept and design, study supervision, critical revision of the manuscript for important intellectual content.

\section{Acknowledgment}

The authors thank the families for their participation; Dr. Elise Heon, Toronto, for facilitating collaboration and providing comments; and Associazione Italiana Lafora for support.

\section{Study funding}

This work was supported by the Ontario Brain Institute, Foundation Fighting Blindness USA, and the National Institute of Neurological Disorders and Stroke of the NIH under award number P01 NS097197. B.A.M. holds the Jimmy Elizabeth Wescott Chair in Pediatric Neurology at the University of Texas Southwestern.

\section{Disclosure}

The authors report no disclosures relevant to the manuscript. Go to Neurology.org/N for full disclosures.

Received November 28, 2017. Accepted in final form April 5, 2018.

\section{References}

1. Andrade DM, Ackerley CA, Minett TS, et al. Skin biopsy in Lafora disease: genotypephenotype correlations and diagnostic pitfalls. Neurology 2003;61:1611-1614.

2. Berard-Badier M, Pellissier JF, Gambarelli D, de Barsy T, Roger J, Toga M. The retina in Lafora disease: light and electron microscopy. Albrecht Von Graefes Arch Klin Exp Ophthalmol 1980;212:285-294.

3. Van Heycop Ten Ham M. Lafora disease: a form of progressive myoclonus epilepsy. In: Magnus WOC, Haas AMLd, eds. The Epilepsies. Amsterdam, NY: North Holland Pub. Co.; American Elsevier; 1974: xiv, 860 p.

4. de Graaf AS, Ancker E, Rutherfoord GS, van der Walt JJ, Rossouw DJ. Lafora-body disease with optic atrophy, macular degeneration and cardiac failure. J Neurol Sci 1989;93:69-84.

5. Pinto WB, de Souza PV, Pinheiro JR, Okamoto KY, Enokihara MM, Oliveira AS. Retinitis pigmentosa in Lafora disease: expanding findings of progressive myoclonic epilepsy. Neurology 2015;85:1087.

6. Carpenter S, de Rezende Pinto WB, de Souza PV, Oliveira AS. Retinitis pigmentosa in Lafora disease: expanding findings of progressive myoclonic epilepsy. Neurology 2016;86:1563.

7. Korczyn AD, Ben-Tovim N. Electroretinographic responses in Lafora disease. Elec troencephalogr Clin Neurophysiol 1978;45:785-788. 